\title{
Dietary proteins improve endothelial function under fasting conditions but not in the postprandial state, with no effects on markers of low-grade inflammation
}

\author{
Karianna F. M. Teunissen-Beekman ${ }^{1,2}$, Janneke Dopheide ${ }^{1,2}$, Johanna M. Geleijnse ${ }^{1,3}$, \\ Stephan J. L. Bakker ${ }^{1,4}$, Elizabeth J. Brink ${ }^{1}$, Peter W. de Leeuw ${ }^{5}$, Casper G. Schalkwijk ${ }^{5}$ and \\ Marleen A. van Baak $^{1,2 *}$ \\ ${ }^{1}$ Top Institute Food and Nutrition, 6700 AN Wageningen, The Netherlands \\ ${ }^{2}$ Department of Human Biology, NUTRIM School for Nutrition, Toxicology and Metabolism, Maastricht University, 6200 MD \\ Maastricht, The Netherlands \\ ${ }^{3}$ Division of Human Nutrition, Wageningen University, 6700 EV Wageningen, The Netherlands \\ ${ }^{4}$ Department of Internal Medicine, University Medical Center Groningen and University of Groningen, 9700 RB Groningen, \\ The Netherlands \\ ${ }^{5}$ Department of Internal Medicine, Maastricht University Medical Center and Cardiovascular Research Institute Maastricht \\ (CARIM), 6200 MD Maastricht, The Netherlands
}

(Submitted 16 April 2015 - Final revision received 30 June 2015 - Accepted 12 August 2015 - First published online 24 September 2015)

\section{Abstract}

Endothelial dysfunction (ED) and low-grade inflammation (LGI) have a role in the development of CVD. The two studies reported here explored the effects of dietary proteins and carbohydrates on markers of ED and LGI in overweight/obese individuals with untreated elevated blood pressure. In the first study, fifty-two participants consumed a protein mix or maltodextrin $(3 \times 20 \mathrm{~g} / \mathrm{d})$ for 4 weeks. Fasting levels and $12 \mathrm{~h}$ postprandial responses of markers of ED (soluble intercellular adhesion molecule 1 (sICAM), soluble vascular cell adhesion molecule 1 (sVCAM), soluble endothelial selectin and von Willebrand factor) and markers of LGI (serum amyloid A, C-reactive protein and sICAM) were evaluated before and after intervention. Biomarkers were also combined into mean $Z$-scores of ED and LGI. The second study compared $4 \mathrm{~h}$ postprandial responses of ED and LGI markers in forty-eight participants after ingestion of $0.6 \mathrm{~g} / \mathrm{kg}$ pea protein, milk protein and egg-white protein. In addition, postprandial responses after maltodextrin intake were compared with a protein mix and sucrose. The first study showed significantly lower fasting ED $Z$-scores and sICAM after 4 weeks on the high-protein diet $(P \leq 0 \cdot 02)$. The postprandial studies found no clear differences of ED and LGI between test meals. However, postprandial sVCAM decreased more after the protein mix compared with maltodextrin in both studies $(P \leq 0 \cdot 04)$. In conclusion, dietary protein is beneficial for fasting ED, but not for fasting LGI, after 4 weeks of supplementation. On the basis of $Z$-scores, postprandial ED and LGI were not differentially affected by protein sources or carbohydrates.

\section{Key words: Proteins: Carbohydrates: Endothelial function: Inflammation: (Pre)hypertension}

Consumption of dietary proteins at the expense of carbohydrates lowers blood pressure ${ }^{(1,2)}$, and mechanisms through which proteins affect blood pressure need to be clarified. Endothelial dysfunction (ED) and low-grade inflammation (LGI) are important factors in the progression of $\mathrm{CVD}^{(3,4)}$, and they are associated with hypertension ${ }^{(4,5)}$. Both macronutrient and micronutrient intake have been found to affect the levels of biomarkers of $\mathrm{ED}$ and $\mathrm{LGI}^{(6,7)}$. However, thus far, no clear effects of long-term increased protein intake on levels of C-reactive protein $(\mathrm{CRP})^{(8-12)}, \mathrm{IL}-6, \mathrm{TNF} \alpha^{(8-11)}$, soluble E-selectin
(sE-selectin), soluble intercellular adhesion molecule 1 (sICAM) and soluble vascular cell adhesion molecule $1(\mathrm{sVCAM})^{(9,10)}$ have been found.

Although the effects of high-energy high-fat meals on postprandial levels of markers of ED and LGI have been studied frequently ${ }^{(13-15)}$, effects of protein and carbohydrate intake on postprandial changes in markers of ED and LGI have been investigated less often. In healthy volunteers, glucose intake was found to increase sICAM, sVCAM, sE-selectin, IL-6 and CRP postprandially compared with fasting values ${ }^{(16)}$. Others found

Abbreviations: CRP, C-reactive protein; ED, endothelial dysfunction; LGI, low-grade inflammation; PROPRES, randomised clinical trial on the effects of PROteins on blood PRESsure; SAA, serum amyloid A; sE-selectin, soluble endothelial selectin; sICAM, soluble intercellular adhesion molecule 1; sVCAM, soluble vascular cell adhesion molecule 1; vWf, von Willebrand factor.

*Corresponding author: M. A. van Baak, fax +31 433670 976, email m.vanbaak@maastrichtuniversity.nl 
no effect of carbohydrate intake on postprandial levels of sICAM, sVCAM, IL-6 ${ }^{(17,18)}$, CRP and sE-selectin ${ }^{(18)}$. With regard to dietary proteins, postprandial responses of markers of ED and LGI to protein intake did not differ from those after carbohydrate intake $^{(19)}$ or placebo ${ }^{(10)}$.

We previously reported a blood-pressure-lowering effect after a 4-week consumption of a protein mix compared with maltodextrin in the randomised clinical trial on the effects of PROteins on blood PRESsure (PROPRES) ${ }^{(20)}$. The present study aimed to explore chronic and postprandial effects of protein intake compared with carbohydrate intake on ED and LGI. We hypothesised that fasting levels of markers of ED and LGI would be lower after 4 weeks on the high-protein diet compared with the high-maltodextrin diet in the PROPRES study and thus might contribute to the lower blood pressure reported earlier ${ }^{(20)}$. To determine whether possible chronic effects of dietary protein on ED and LGI can also be observed acutely, we compared postprandial levels of markers of ED and LGI after ingestion of protein- and maltodextrin-supplemented meals on the first and last day of the intervention in the PROPRES trial. A second postprandial study was conducted to investigate whether different types of proteins or carbohydrates would differentially affect postprandial responses of ED and LGI.

\section{Methods}

\section{Study population}

The first study was conducted in the year 2009 in a subset of the study participants of the PROPRES study $\left(\begin{array}{l}n \\ 52\end{array}\right)^{(21)}$, and it consisted of a chronic, 4-week intervention, and postprandial studies with postprandial assessments on the first and last day of the intervention (online Supplementary Fig. S1). Participants in the chronic and postprandial parts of the PROPRES study were overweight and obese individuals with untreated elevated blood pressure (systolic blood pressure of 130-159 $\mathrm{mmHg}$ and/ or diastolic blood pressure of $85-99 \mathrm{mmHg}$ ). More information of inclusion and exclusion criteria are described elsewhere ${ }^{(20)}$. The second study was conducted in the year 2011, and it consisted of a crossover evaluation of postprandial effects of dietary proteins and carbohydrates. Participants had similar characteristics as in the first study, and part of this study group had also participated in the PROPRES study ${ }^{(22)}$. Both the first and second studies were conducted according to the guidelines laid down in the Declaration of Helsinki, and all procedures involving human subjects were approved by the medical-ethical committee of Maastricht University Medical Center and Maastricht University (METC azM/UM). Written informed consent was obtained from all participants.

\section{Chronic study}

The chronic part of the PROPRES trial had a randomised parallel group design and compared the effects of 4 weeks of exchanging $3 \times 20 \mathrm{~g} / \mathrm{d}$ of dietary carbohydrates isoenergetically with a protein mixture (20\% pea protein, $20 \%$ soya protein, $30 \%$ eggwhite protein, $30 \%$ milk protein isolates) or with maltodextrin on ED and LGI. More details on the 4-week PROPRES trial can be found elsewhere ${ }^{(20)}$.

\section{First postprandial study}

This study was also part of the PROPRES trial. Twelve-hour postprandial responses to standard mixed meals isoenergetically supplemented with $20 \mathrm{~g}$ of the protein mixture or with $20 \mathrm{~g}$ of maltodextrin were compared during a $12 \mathrm{~h}$ test day at the start and a $12 \mathrm{~h}$ test day at the end of the 4-week PROPRES trial. More details on the postprandial part of the PROPRES study can be found elsewhere ${ }^{(21)}$.

\section{Second postprandial study}

The $4 \mathrm{~h}$ postprandial responses after single ingestion of three types of protein (pea, milk and egg-white protein isolate) and two types of carbohydrate (maltodextrin and sucrose) and the protein mix used in the PROPRES study were assessed in fortyeight overweight participants with untreated elevated blood pressure in the morning after an overnight fast. The six test meals in this study contained $0.6 \mathrm{~g} / \mathrm{kg}$ body mass of one of the test products. Products were tested in a randomised order crossover design (online Supplementary Fig. S2). Comparisons were made between the three protein sources, and responses to the ingestion of maltodextrin were compared with those after the ingestion of sucrose and those after the protein mix. More details of this study are described elsewhere ${ }^{(22)}$.

\section{Measurements}

Because of their relationship with the risk for $\mathrm{CVD}^{(23)}$ and their responsiveness to nutritional interventions ${ }^{(24)}$, von Willebrand factor (vWf), sE-selectin, sICAM, sVCAM, CRP and serum amyloid A (SAA) were chosen as markers of ED (vWf, sE-selectin, sICAM and SVCAM) and LGI (CRP, SAA and SICAM) in these studies.

Chronic study. Venous blood for measurement of markers of ED and LGI was collected before breakfast in the fasting state on the first and last days of the 4-week intervention.

First postprandial study. Postprandial measurements of vWf and sE-selectin were taken at 1, 2 and $4 \mathrm{~h}$ after the protein- or maltodextrin-supplemented breakfast (Fig. 1(a)). sICAM, sVCAM, CRP and SAA were measured every $30 \mathrm{~min}$ for the first $2 \mathrm{~h}$ after breakfast $(30,60,90,120 \mathrm{~min})$ and then hourly for the following $10 \mathrm{~h}$ (3, 4, 5, 6, 7, 8, 9, 10, 11 and $12 \mathrm{~h}$ ) (Fig. 1(a)). Lunch and dinner were consumed 4 and $8 \mathrm{~h}$ after breakfast, respectively. These meals were also supplemented with the protein mix or maltodextrin. More details of the meals are described elsewhere ${ }^{(21)}$.

Second postprandial study. All markers were measured in the fasting state and hourly during $4 \mathrm{~h}$ after the test meal (60, 120, 180 and $240 \mathrm{~min}$ ) (Fig. 1(b)).

Plasma sICAM, sVCAM, sE-selectin, CRP and SAA were assessed using a multi-array detection system based on electro-chemiluminescence technology (SECTOR Imager 2400; 
(a)

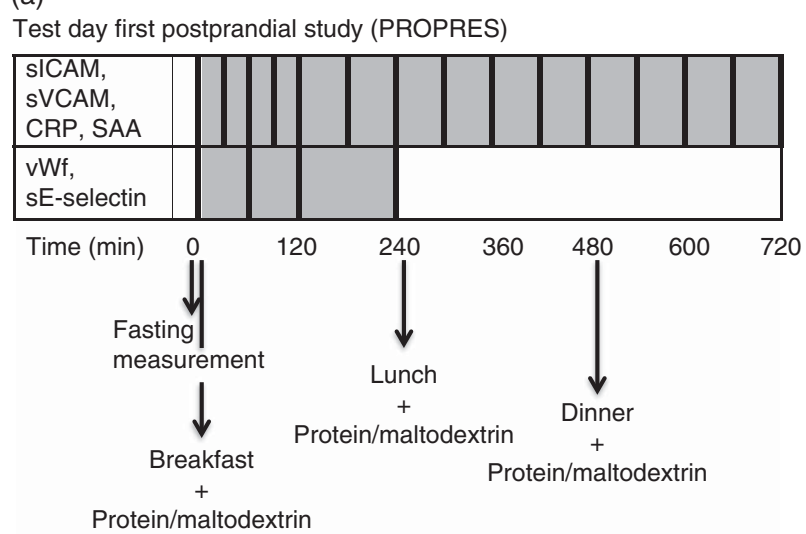

(b)

Test day second postprandial study

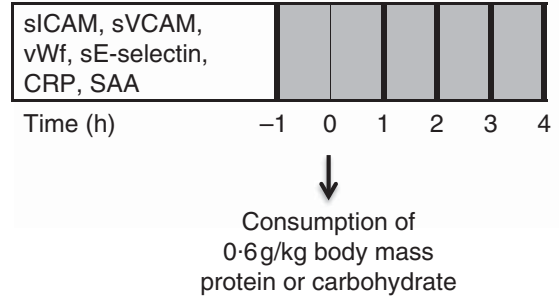

Fig. 1. Measurements on the test days in the first postprandial study (a) and second postprandial study (b). Thick vertical lines represent measurements of parameters mentioned in the same row. CRP, C-reactive protein; PROPRES, randomised clinical trial on the effects of PROteins on blood PRESsure; SAA, serum amyloid A; sE-selectin, soluble endothelial selectin; sICAM, soluble intercellular adhesion molecule 1; sVCAM, soluble vascular cell adhesion molecule 1; vWf, von Willebrand factor.

Meso Scale Discovery), as previously described ${ }^{(25)}$. vWf was assessed in citrated plasma by means of an ELISA ${ }^{(25)}$.

\section{Statistical analyses}

In addition to responses of individual biomarkers, overall $Z$-scores for ED and LGI were also analysed to cope with biological variability of each individual marker ${ }^{(25)}$ and to ease the interpretation of findings for individual biomarkers. To calculate ED and LGI $Z$-scores, first a $Z$ was calculated for each individual biomarker as follows: $Z=$ (individual value-population mean)/ population SD. Individual biomarker $Z$-scores were averaged into the overall $Z$-scores for ED and LGI. The overall ED $Z$-scores consisted of the biomarkers sICAM, sVCAM, sE-selectin and vWf. Overall LGI $Z$-scores consisted of CRP, SAA and sICAM. Overall ED $Z$-scores for the $12 \mathrm{~h}$ test day only included sICAM and sVCAM, because the other markers were not determined after lunch until the end of the test day. Further explanations on the calculation of overall $Z$-scores can be found elsewhere ${ }^{(25)}$. The population mean and population standard deviation for each individual biomarker were calculated as follows in the different studies: for the chronic study the population mean (and standard deviation) was based on the average of the fasting levels on the first and last day of the intervention. For the first postprandial study, the population mean (and standard deviation) was based on the average of all measurements during one test day. For the second postprandial study, the population mean (and standard deviation) was based on the average of all measurements during all test days, because this was a crossover trial.

All statistical analyses were performed with the SPSS software (version 20; IBM). A $P$ value of $<0.05$ was considered to be significant. CRP and SAA were ln-transformed because of their skewed distributions. Participants with CRP values $>10 \mathrm{mg} / \mathrm{l}$ were excluded from analyses, because a CRP value this high indicates the presence of inflammation ${ }^{(26)}$, which could confound the postprandial responses.

Chronic study. Differences in fasting values between groups on day 1 of supplementation were tested with an independent samples $t$ test. Fasting values after 4 weeks were tested with a univariate ANCOVA with the fasting value on day 1 as a covariate.

First postprandial study. Postprandial data on each test day were analysed by a linear mixed model analysis using a random intercept model. Analyses started off with the full model including group, time, fasting measurement, BMI, age and sex. BMI, age and sex were removed from the model one-by-one starting with the least significant covariate to test whether they significantly contributed to the model with a -2 log-likelihood test. Significant covariates were kept in the final model. Incremental AUC (iAUC) were calculated in the PROPRES study and tested for deviance from 0 with a one-sample $t$ test to see whether postprandial increases/decreases were significant.

Second postprandial study. Postprandial responses were analysed with a linear mixed model approach that took the crossover design and the correlation between repeated measures into account. The basic model consisted of a random intercept at the individual level. If significant, this model was extended with a random intercept at the meal within individual level (individual $\times$ meal) or with a serial correlation over time. The basic model always included the following variables: time, meal, fasting measurements and two variables controlling for the crossover design - meal order and test day number. The interaction term between meal and time (meal $\times$ time) and the covariates age, sex and BMI were kept in the model if significant. In case of significant meal $\times$ time or meal effect, five post hoc comparisons were made - that is, maltodextrin $v$. sucrose, maltodextrin $v$, protein mix, pea protein $v$. milk protein, pea protein $v$. egg-white protein and milk protein $v$. eggwhite protein. The critical $P$ value was corrected for these comparisons; therefore, post hoc differences were considered significant at $P \leq 0 \cdot 01^{(22)}$.

\section{Results}

From the fifty-two participants in the chronic study and first postprandial study (maltodextrin group $n$ 27, protein group $n$ 25), three participants in the maltodextrin group were excluded from the present analyses (online Supplementary Fig. S1). This was because two participants had CRP values $>10 \mathrm{mg} / \mathrm{l}$ on one of the test days, and another participant had 
missing fasting values on one test day. In the second postprandial study, product test days were excluded from analyses when a participant had CRP levels $>10 \mathrm{mg} / \mathrm{l}$ during that particular day. As a consequence, one test of sucrose, two tests of pea protein, two tests of milk protein, two tests of egg-white protein and three tests of the protein mix were excluded from analyses (online Supplementary Fig. S2). Baseline characteristics of participants in the first postprandial study and the second postprandial study have been published previously ${ }^{(21,22)}$.

\section{Chronic study: effects of the 4 weeks increased protein intake on endothelial dysfunction and low-grade inflammation}

ED $Z$-scores, sICAM and sE-selectin were significantly lower in the protein group at the start of the intervention $(P \leq 0.04$, Table 1), whereas only baseline-corrected ED $Z$-scores and sICAM were significantly lower in the protein group after 4 weeks of supplementation $(P \leq 0 \cdot 02$, Table 1$)$.

First postprandial study: postprandial responses

of endothelial dysfunction to protein- and maltodextrin-supplemented meals

Analyses of iAUC showed significant postprandial decreases on the first day of supplementation and after 4 weeks of supplementation for ED $Z$-scores, sICAM, sVCAM and sE-selectin $(P \leq 0 \cdot 05)$. Postprandial vWf decreased below fasting values on day 1 of supplementation $(P \leq 0 \cdot 05)$, but not after 4 weeks.

Twelve-hour postprandial levels of ED $Z$-scores (composed of SICAM and SVCAM) and SICAM tended to be lower after ingestion of the protein-supplemented meals than after ingestion of the maltodextrin-supplemented meals on day 1 ( $P=0.07,0 \cdot 08$, respectively; Fig. 2(a) and (c)), but these did not differ significantly after 4 weeks (Fig. 2(b) and (d)). Postprandial sVCAM levels were significantly lower after ingestion of the protein-supplemented meals compared with the maltodextrinsupplemented meals after 4 weeks of supplementation $(P=0.04$, Fig. 2(f)), but not on the first day of supplementation (Fig. 2(e)). Four-hour postprandial responses of ED $Z$-scores (composed of sICAM, sVCAM, sE-selectin and vWf), sE-selectin and vWf did not differ between protein- and maltodextrinsupplemented meals on both test days (data not shown).

\section{First postprandial study: postprandial responses \\ of low-grade inflammation to protein- and maltodextrin-supplemented meals}

Analyses of iAUC revealed significant postprandial decreases of LGI $Z$-scores (composed of CRP, SAA and sICAM) on both test days (iAUC $P \leq 0 \cdot 05$, Fig. 3(a) and (b)), whereas postprandial CRP levels increased significantly on day 1 and postprandial SAA levels decreased significantly after 4 weeks (iAUC $P \leq 0 \cdot 05$, Fig. 3(c) and (f), respectively). Postprandial responses of LGI $Z$-scores, CRP and SAA did not differ between protein- and maltodextrin-supplemented meals (Fig. 3(a)-(f)).

\section{Second postprandial study: postprandial responses of endothelial dysfunction after consumption of various protein and carbohydrate sources}

Postprandial levels of ED $Z$-scores did not differ between protein sources or between maltodextrin $v$. sucrose or maltodextrin $v$. protein mix (Fig. 4(a) and (b)). Pea protein consumption induced lower postprandial levels of sICAM compared with egg-white protein consumption $(P \leq 0.001)$. Ingestion of the protein mix resulted in lower postprandial levels of sICAM and sVCAM compared with ingestion of maltodextrin $(P \leq 0.004$, Fig. 4(c)-(f)). Postprandial responses of sE-selectin and vWf did not differ between test meals (Fig. 4(g)-(j)).

\section{Second postprandial study: postprandial responses of LGI after consumption of various protein and carbohydrate sources}

Postprandial responses in LGI $Z$-scores did not differ between protein sources or between maltodextrin and sucrose or maltodextrin and the protein mix (Fig. 5(a) and (b)). Ingestion of pea protein induced higher postprandial SAA levels than

Table 1. Baseline and 4-week levels of markers of endothelial dysfunction (ED) and low-grade inflammation (LGI) in the chronic study (Mean values with their standard errors)

\begin{tabular}{|c|c|c|c|c|c|c|c|c|c|c|}
\hline & \multicolumn{4}{|c|}{ Maltodextrin } & \multirow[b]{3}{*}{$n$} & \multicolumn{4}{|c|}{ Protein } & \multirow[b]{3}{*}{$n$} \\
\hline & \multicolumn{2}{|c|}{ Day 1} & \multicolumn{2}{|c|}{4 weeks } & & \multicolumn{2}{|c|}{ Day 1} & \multicolumn{2}{|c|}{4 weeks } & \\
\hline & Mean & SEM & Mean & SEM & & Mean & SEM & Mean & SEM & \\
\hline ED Z-score & $0.30^{*}$ & 0.18 & $0.27 \dagger$ & 0.15 & 21 & $-0.21^{\star}$ & 0.12 & $-0.33 \dagger$ & 0.10 & 22 \\
\hline LGI Z-score & -0.01 & 0.15 & 0.24 & 0.16 & 24 & -0.14 & 0.18 & -0.08 & 0.14 & 25 \\
\hline $\operatorname{sICAM}(\mu \mathrm{g} / \mathrm{l})$ & $215^{\star}$ & 8 & $224 \dagger$ & 8 & 24 & $191^{*}$ & 7 & $182 \dagger$ & 6 & 25 \\
\hline sVCAM $(\mu \mathrm{g} / \mathrm{l})$ & 362 & 19 & 365 & 17 & 24 & 353 & 13 & 351 & 13 & 25 \\
\hline sE-selectin $(\mu \mathrm{g} / \mathrm{l})$ & $100^{*}$ & 7 & 101 & 8 & 21 & $73^{*}$ & 7 & 69 & 6 & 22 \\
\hline vWf (\%) & 102 & 7 & 92 & 6 & 21 & 86 & 6 & 86 & 6 & 22 \\
\hline $\mathrm{CRP}(\mathrm{mg} / \mathrm{l}) \ddagger$ & 1.79 & 0.35 & $2 \cdot 29$ & 0.45 & 24 & 1.51 & 0.30 & 1.50 & 0.20 & 25 \\
\hline SAA $(\mathrm{mg} / \mathrm{l}) \ddagger$ & $1 \cdot 17$ & 0.23 & 1.48 & 0.28 & 24 & 1.65 & 0.34 & 1.77 & 0.23 & 25 \\
\hline
\end{tabular}

CRP, C-reactive protein; SAA, serum amyloid A; sE-selectin, soluble endothelial selectin; sICAM, soluble intercellular adhesion molecule 1; sVCAM, soluble vascular cell adhesion molecule 1; vWf, von Willebrand factor.

${ }^{*} P<0.05$ for between-group differences on day 1 tested with an independent samples $t$ test.

$\dagger P<0.05$ for between-group differences after 4 weeks tested with ANCOVA correcting for the fasting value on day 1.

$\ddagger$ Statistical tests were performed with In-transformed data. 
(a)

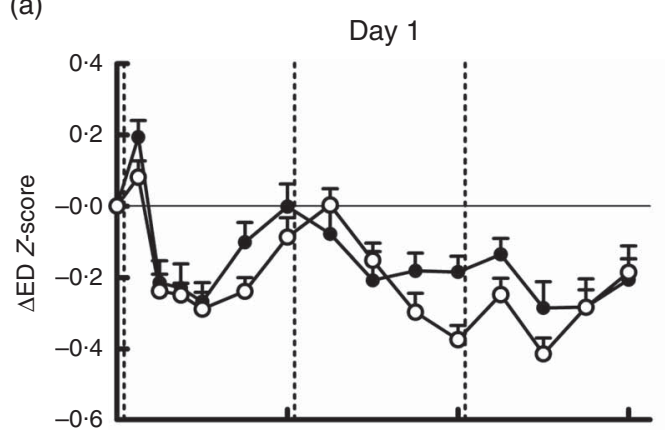

(c)

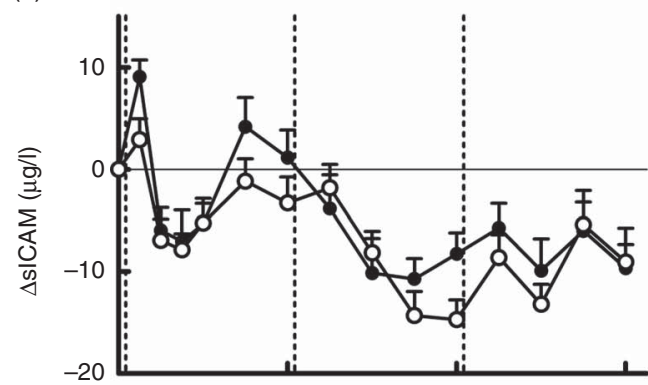

(e)

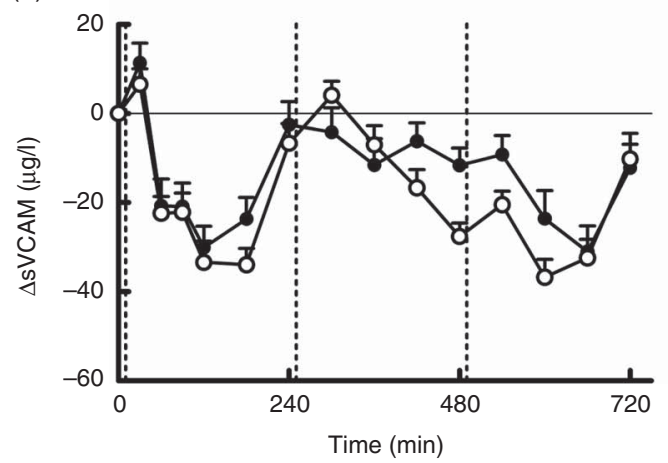

(b)

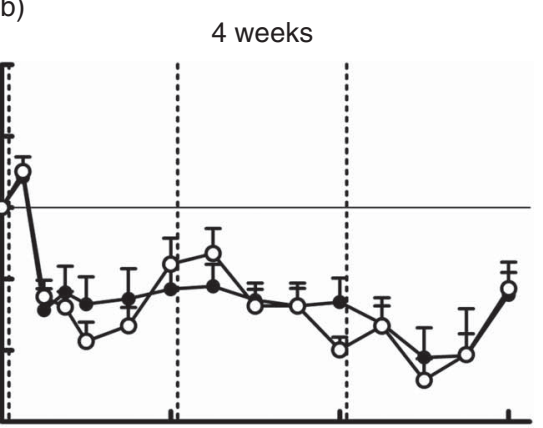

(d)

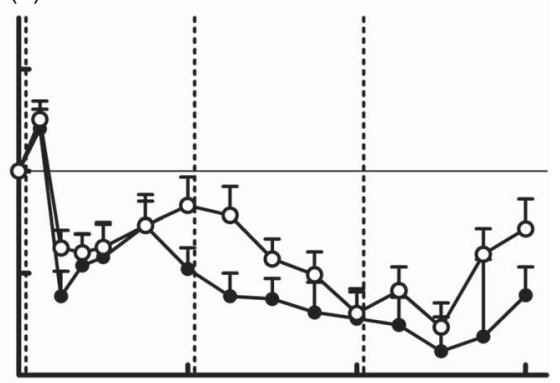

(f)

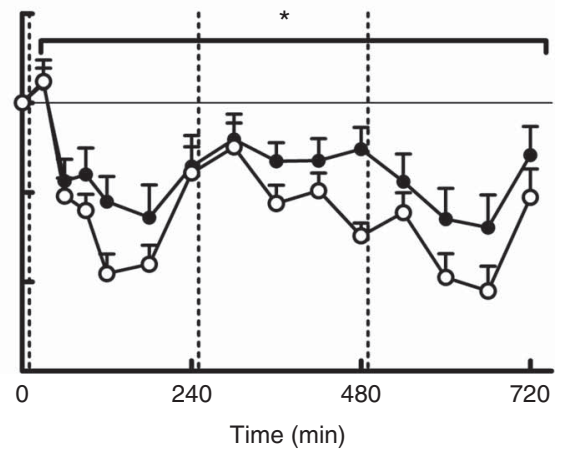

Fig. 2. $12 \mathrm{~h}$ Postprandial responses of endothelium function to meals supplemented with protein or maltodextrin on day 1 of supplementation (left) and after 4 weeks of supplementation (right) in the first postprandial study. (a, b) Endothelial dysfunction (ED) Z-scores, (c, d) soluble intercellular adhesion molecule 1 (sICAM) and (e, f) soluble vascular cell adhesion molecule 1 (sVCAM). Participants included in analyses: maltodextrin group $(-, n 24)$ and protein group ( $-0-n 25)$. Vertical lines indicate breakfast, lunch and dinner. Values are means with their standard errors. ${ }^{*} P<0.05$ for the difference between the maltodextrin group and the protein group over the whole $12 \mathrm{~h}$ period according to the mixed model.

ingestion of milk and egg-white protein $(P<0 \cdot 001$, Fig. 5(e) and (f)). Ingestion of the protein mix resulted in higher postprandial CRP levels than ingestion of maltodextrin $(P<0.001$, Fig. 5(c) and (d)).

\section{Discussion}

\section{Chronic study}

The most important findings in the studies reported here are the lower fasting levels of ED $Z$-scores and sICAM after 4 weeks on the protein diet compared with the maltodextrin diet in the PROPRES study. Literature data on the effects of proteinenriched diets on fasting levels of markers of ED are relatively scarce. Two-week consumption of a novel wheyderived peptide $(5 \mathrm{~g} / \mathrm{d})$ did not affect fasting values of
sE-selectin, sICAM, sVCAM and CRP compared with placebo in healthy individuals ${ }^{(10)}$. Eight-week consumption of $40 \mathrm{~g} / \mathrm{d}$ soyabean protein (including isoflavones) lowered fasting levels of sE-selectin compared with intake of $40 \mathrm{~g} / \mathrm{d}$ milk protein, but not compared with the intake of $40 \mathrm{~g} / \mathrm{d}$ complex carbohydrate. No differences between supplements were found for fasting sICAM and sVCAM levels ${ }^{(9)}$. In contrast to these two studies, we demonstrated a decrease of sICAM levels after the protein mix. One explanation for our finding could be the greater difference in protein intake $(60 \mathrm{~g} / \mathrm{d})$ between groups in our study. In addition to soya and milk protein, our protein mix also consisted of pea protein and egg-white protein. Therefore, our results are consistent with the view that increased protein intake improves markers of ED within 4 weeks under fasting conditions. This may be related to the dose of protein and/or the mix of protein sources that were tested. 
(a)

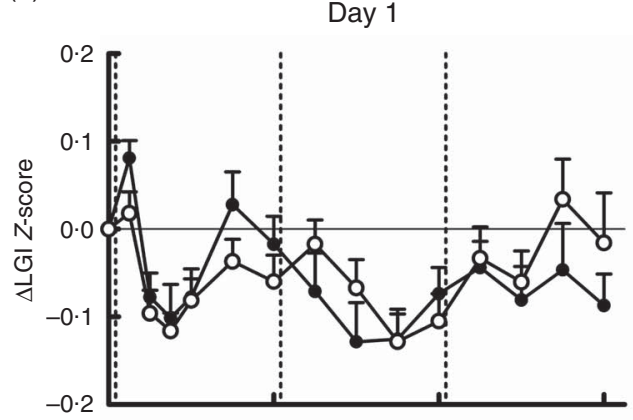

(c)

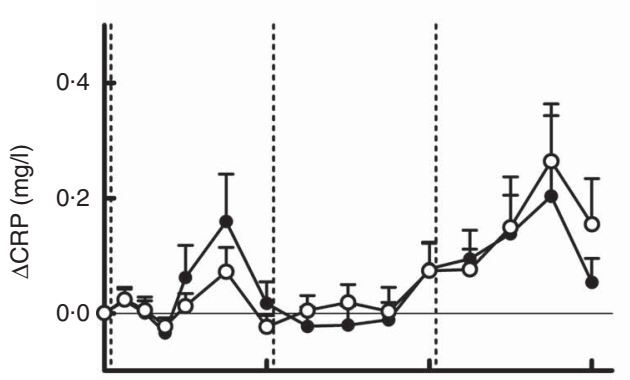

(e)

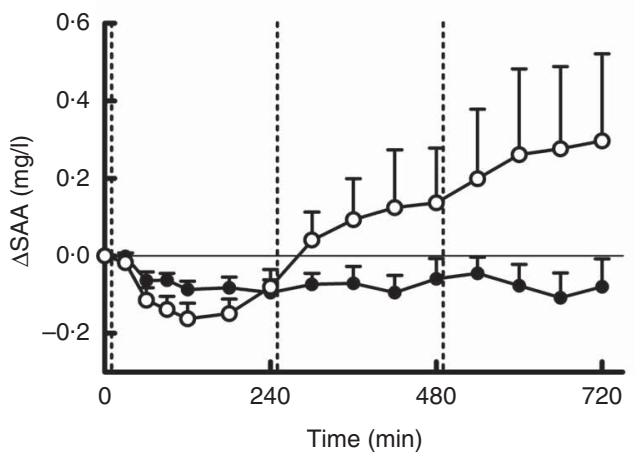

(b)

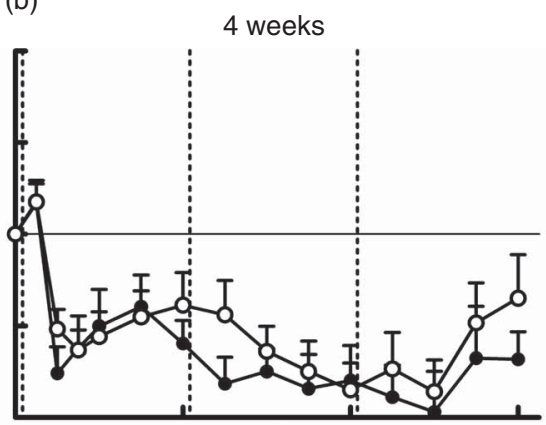

(d)

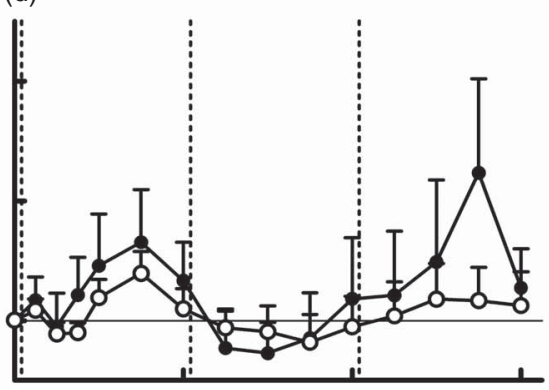

(f)

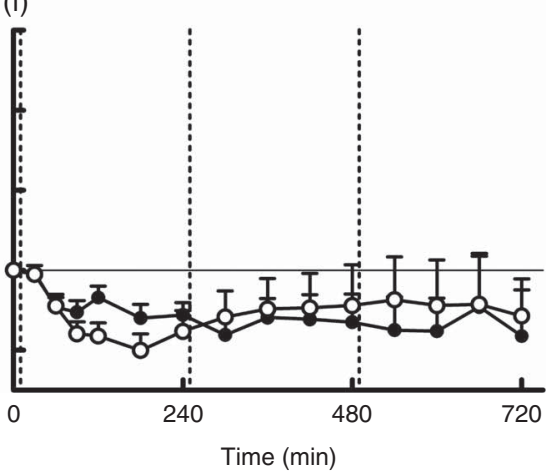

Fig. 3. $12 \mathrm{~h}$ Postprandial responses of low-grade inflammation (LGI) to meals supplemented with protein or maltodextrin on day 1 of supplementation (left) and after 4 weeks of supplementation (right) in the first postprandial study. (a, b) LGI Z-scores, (c, d) C-reactive protein (CRP) and (e, f) serum amyloid A (SAA). Participants included in analyses: maltodextrin group $(-\ldots, n 24)$ and protein group $\left(-C_{-}, n 25\right)$. Vertical lines indicate breakfast, lunch and dinner. Values are means with their standard errors. Postprandial responses did not differ between groups according to the mixed model.

Fasting levels of markers of LGI did not differ between the protein and maltodextrin group after the 4-week intervention. This is consistent with a 12 week trial, which found no differences in fasting concentrations of TNF $\alpha$, IL-6 and CRP between a whey-, casein- or glucose-supplemented diet $(54 \mathrm{~g} / \mathrm{d})^{(11)}$. In another study, fasting CRP levels did not significantly differ after 8 weeks of soyabean protein or milk protein consumption compared with complex carbohydrate ${ }^{(9)}$. Eight weeks on a high-protein weight loss diet (30\% of energy intake) also did not affect fasting CRP levels compared with a highcarbohydrate/fibre weight loss diet (20\% of energy intake from protein) ${ }^{(12)}$, nor did 1-week consumption of milk tripeptides $v$. placebo $^{(27)}$. However, two other studies found unfavourable effects of increased protein intake on LGI. The DIOGENES randomised controlled trial, comparing diets with higher/ lower-protein content combined with higher/lower glycaemic index, found evidence that the lower-protein diets were associated with lower fasting CRP concentrations than the higherprotein diets ${ }^{(28)}$. A recent weight loss trial found increased inflammation scores after a high-protein weight loss diet $(30 \%$ of energy intake) compared with a normal-protein weight loss diet ( $15 \%$ of energy intake), whereas levels of individual markers CRP, TNF $\alpha$, plasminogen activator inhibitor-1 (PAI-1) and IL- 6 did not differ between groups. This increased inflammation score was found especially for high animal and meat protein intake and not for increased vegetable or fish protein intake $^{(8)}$. The protein supplement in our study was only partly composed of animal protein (60\%:30\% milk and 30\% eggwhite protein) and not in the form of meat protein, which may explain why we did not find increased LGI in the protein group. In addition, the LGI $Z$-score in our study was composed of other markers (CRP, SAA and sICAM $v$. CRP, TNF $\alpha$, PAI- 1 and IL-6) ${ }^{(8)}$. 
(a)

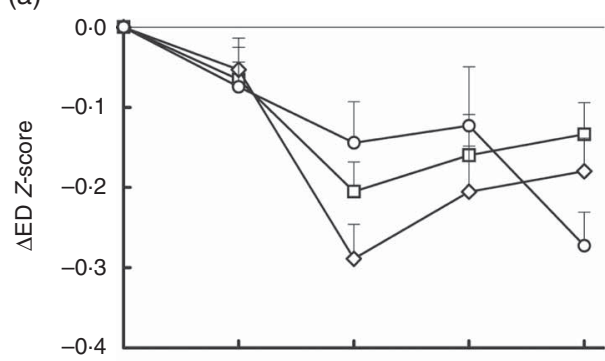

(c)

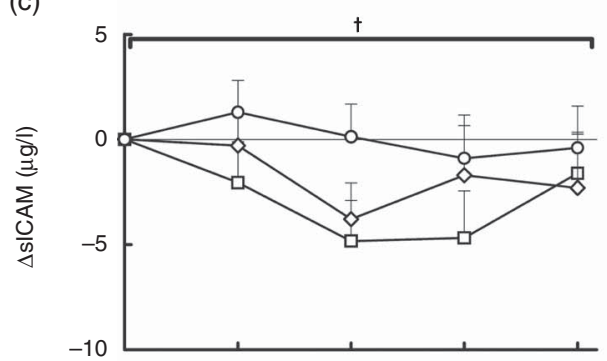

(e)

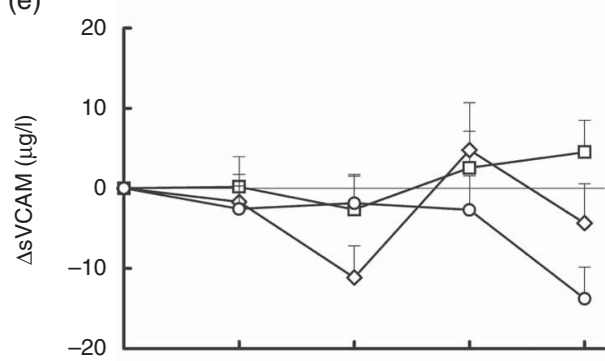

(g)

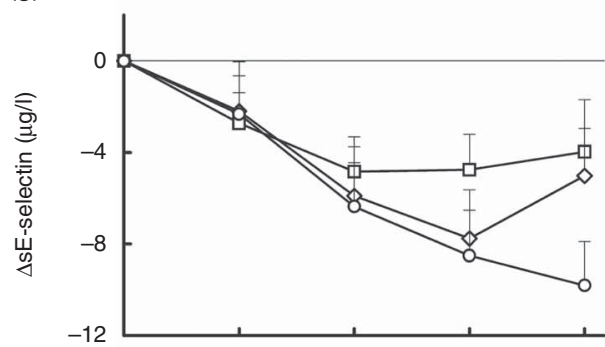

(i)

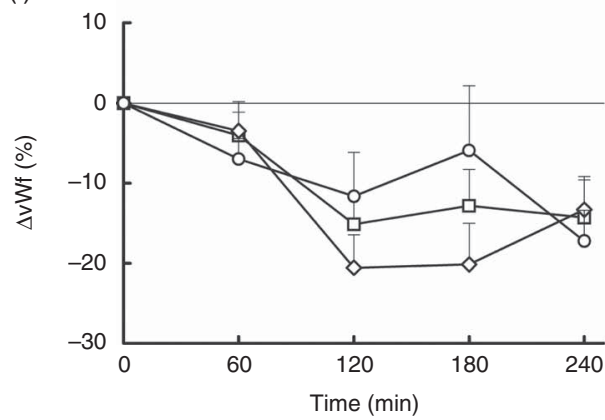

(b)

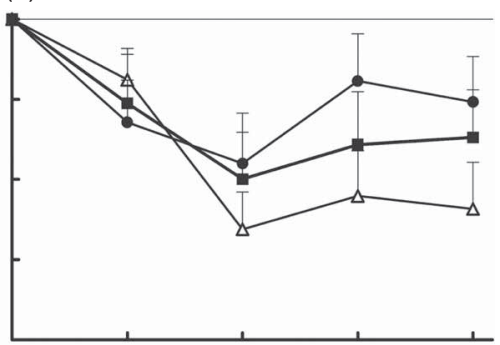

(d)

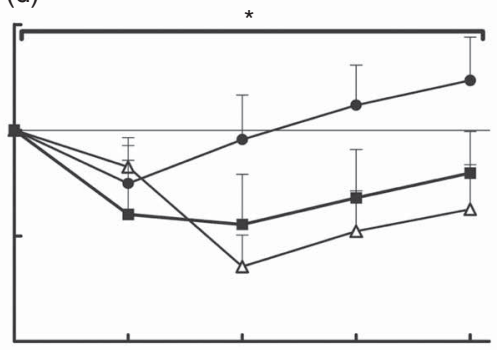

(f)

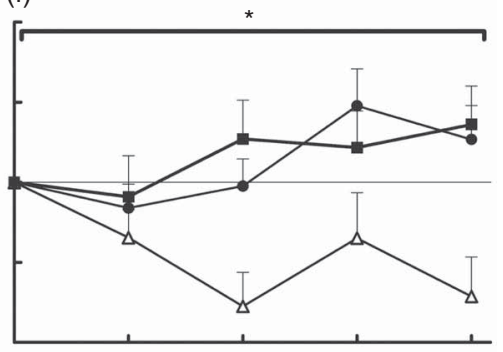

(h)

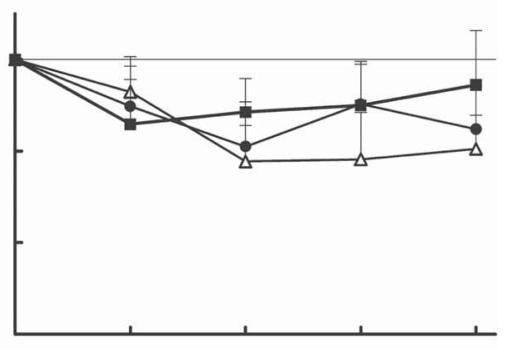

(j)

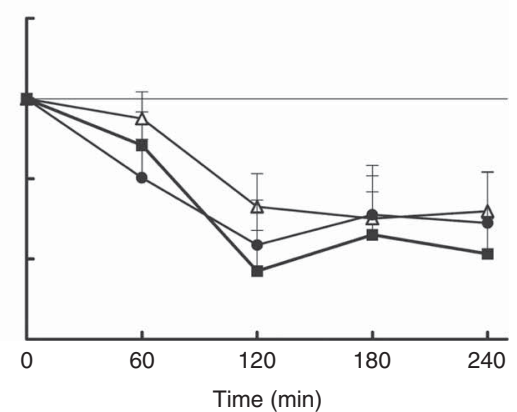

Fig. 4. $4 \mathrm{~h}$ Postprandial responses of endothelium function to different protein sources (left) and maltodextrin, sucrose and a protein mix (right) in the second postprandial study. (a, b) Endothelial dysfunction (ED) Z-scores, (c, d) soluble intercellular adhesion molecule 1 (sICAM), (e, f) soluble vascular cell adhesion molecule 1 (sVCAM), $(\mathrm{g}, \mathrm{h})$ soluble endothelial selectin (sE-selectin) and (i, j) von Willebrand factor (vWf). Participants included in analyses: pea protein ( $\square-, n$ 45), milk

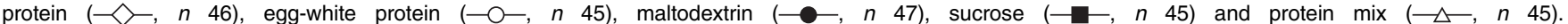
Values are means with their standard errors. * Significant difference between protein mix and maltodextrin over the whole $4 \mathrm{~h}$ period shown by post hoc tests with Bonferroni correction $(P \leq 0.01)$ if the meal was significant $(P \leq 0.05)$. + Significant difference between pea protein and egg-white protein over the whole $4 \mathrm{~h}$ period shown by post hoc tests with Bonferroni correction $(P \leq 0.01)$ if the meal was significant $(P \leq 0.05)$. 
(a)

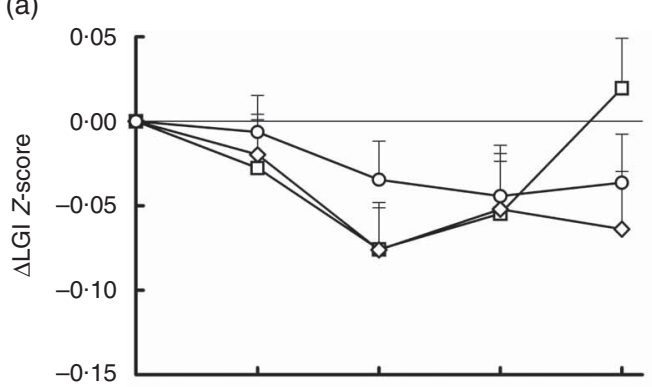

(c)

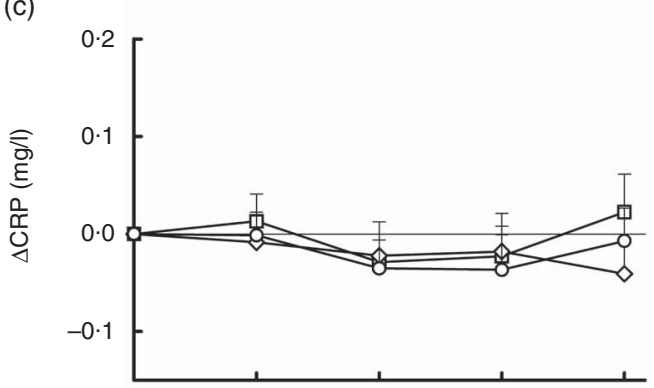

(e)

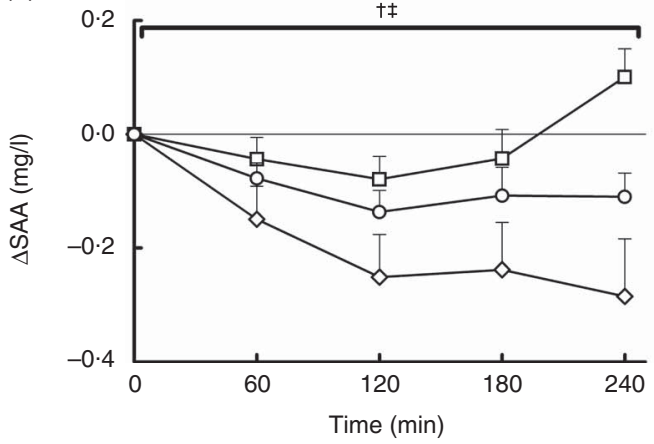

(b)

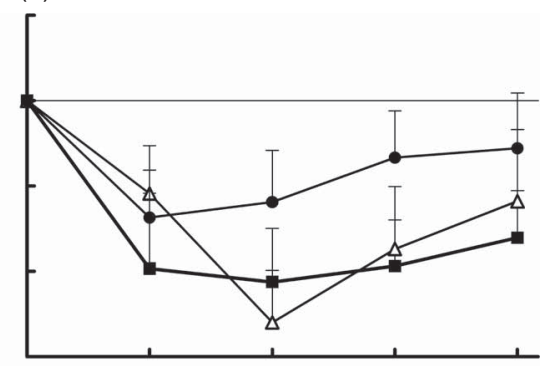

(d)

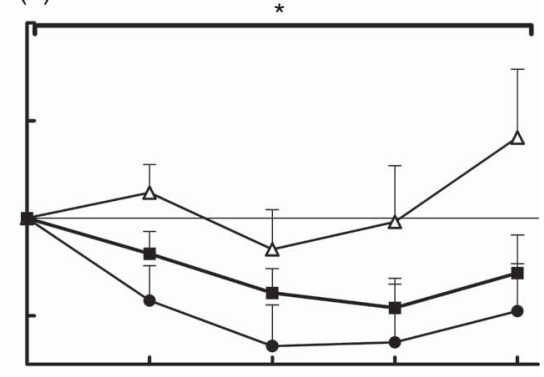

(f)

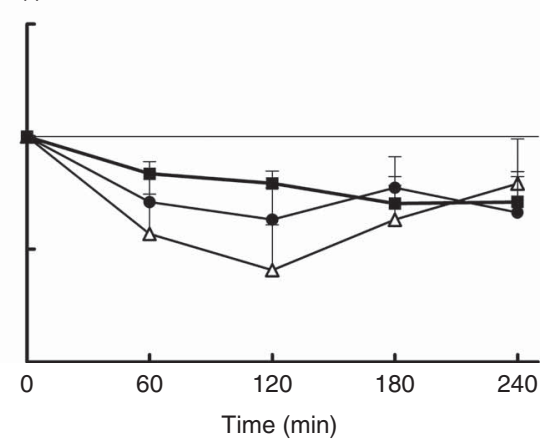

Fig. 5. $4 \mathrm{~h}$ Postprandial responses of low-grade inflammation (LGI) to different protein sources (left) and maltodextrin, sucrose and a protein mix (right) in the second postprandial study. (a, b) LGI Z-scores, (c, d) C-reactive protein (CRP) (e, f) and serum amyloid A (SAA). Participants included in analyses: pea protein ( $\square-\square, n 45)$, milk protein $(-, n 46)$, egg-white protein $(-\bigcirc, n 45)$, maltodextrin $(-\infty, n 47)$, sucrose $(-\square, n 45)$ and protein mix $(-\triangle-n 45)$. Values are means with their standard errors. * Significant difference between protein mix and maltodextrin over the whole $4 \mathrm{~h}$ period shown by post hoc tests with Bonferroni correction $(P \leq 0.01)$ if a meal was significant $(P \leq 0.05)$. † Significant difference between pea protein and egg-white protein over the whole $4 \mathrm{~h}$ period shown by post hoc tests with Bonferroni correction $(P \leq 0.01)$ if meal was significant $(P \leq 0.05)$. $\ddagger$ Significant difference between pea protein and milk protein over the whole $4 \mathrm{~h}$ period shown by post hoc tests with Bonferroni correction $(P \leq 0.01)$ if meal was significant $(P \leq 0.05)$.

Thus, we may conclude that increased protein intake has no effect on the levels of markers of LGI under fasting conditions, which is in agreement with several studies ${ }^{(9,12,19,27)}$ but contrasts with findings of two food-based interventions ${ }^{(8,28)}$. This may be related to differences in the type of proteins included in the diets/supplements. Moreover, in food-based studies, the effects of other food constituents cannot be fully excluded.

\section{First and second postprandial studies}

Postprandial sVCAM levels were found to be lower after protein-supplemented meals compared with maltodextrinsupplemented meals after 4 weeks of intervention in the first postprandial study and after consumption of $0.6 \mathrm{~g} / \mathrm{kg}$ of the protein mix compared with maltodextrin in the second postprandial study. Glucose and insulin might be involved in this difference. In a study investigating the effects of ingestion of $75 \mathrm{~g}$ glucose $v$. fructose, both carbohydrates induced similar $3 \mathrm{~h}$ postprandial responses of sVCAM despite different postprandial glucose and insulin responses ${ }^{(18)}$, which suggests that glucose and insulin do not modulate postprandial sVCAM levels. We found that other markers of ED and the ED $Z$-scores did not differ consistently between the protein mix and maltodextrin. Taken together, we found no clear differences in postprandial responses of ED between a protein mix and maltodextrin.

Postprandial CRP levels were found to be significantly higher after ingestion of the protein mix than after ingestion of maltodextrin in the second postprandial study. However, this difference is unlikely to be detrimental, as baseline CRP levels were low and the difference in postprandial CRP was small. In addition, postprandial levels of LGI $Z$-scores, SAA and sICAM did not differ after ingestion of the protein mix compared with maltodextrin. In 
addition, in our first postprandial study, we did not find significant differences in postprandial levels of LGI markers between the protein- and maltodextrin-supplemented meals, which is in accordance with another study comparing postprandial effects of protein and carbohydrate-supplemented breakfasts ${ }^{(19)}$. In conclusion, we found no clear evidence of differences in postprandial LGI between a protein mix and maltodextrin.

Postprandial responses of markers of ED and LGI were also compared between pea protein, milk protein and egg-white protein ingestion in the second postprandial study. Postprandial sICAM levels were lower after pea protein compared with eggwhite protein. The higher arginine content in pea protein (8.7/ $100 \mathrm{~g})$ compared with milk protein $(3 \cdot 7 / 100 \mathrm{~g})$ and egg-white protein $(6 / 100 \mathrm{~g})$ might be involved in this, as arginine has been suggested to improve endothelial function because of its role in nitric oxide synthesis ${ }^{(29)}$. Addition of arginine to amino acid meals ${ }^{(30)}$ or high-fat meals ${ }^{(31)}$ was shown not to affect postprandial levels of $\operatorname{sICAM}^{(30)}$ or $\mathrm{vWf}^{(30,31)}$. We found that pea protein induced higher postprandial SAA levels compared with egg-white protein and milk protein. Holmer-Jensen et al. ${ }^{(32)}$ also found differences in postprandial inflammation between high-fat mixed meals supplemented with different protein sources. Whey protein consumption, which was the most insulinotropic protein source, resulted in lower postprandial CC chemokine ligand -5 concentrations but the highest postprandial levels of monocyte chemotactic protein 1. Both are markers of inflammation, and the authors could not explain the opposing effects of whey protein ${ }^{(32)}$. In our study, the most insulinotropic protein (pea protein ${ }^{(22)}$ ) also showed opposing effects on postprandial responses of LGI markers (lower sICAM and higher SAA levels) compared with the other protein sources. Whether postprandial insulin levels have a role in postprandial levels of sICAM and SAA remains unclear.

Some limitations need to be considered when interpreting the results of the studies reported in this paper. One limitation is that measurements discussed in this paper were not the primary end points of these studies. Therefore, these studies could be underpowered for finding significant differences in ED and LGI between groups. In addition, only few of the currently available biomarkers of ED and LGI were measured in these studies. Therefore, the effects of dietary proteins on ED and LGI need to be explored further by studying other biomarkers of ED and LGI in addition to the biomarkers measured in our studies such as endothelin-1, nitric oxide metabolites, PG, TNF $\alpha$ and IL- $6{ }^{(3)}$. Another limitation of our studies is the lack of a functional measurement of ED, such as flow-mediated dilation (FMD). This was not manageable because of the large number of measurements taken in both studies ${ }^{(21,22)}$. However, the addition of FMD does not improve the association of circulating biomarkers with carotid arterial stiffening ${ }^{(33)}$. Finally, the role of isoflavones in the improvement of markers of ED in the chronic study cannot be fully excluded ${ }^{(34)}$, because isoflavones could have been present in the soya protein isolate in the protein mix. In conclusion, the present study found evidence that fasting ED $Z$-scores and sICAM levels are lower after 4 weeks on an increased protein diet compared with an increased maltodextrin diet, whereas fasting levels of biomarkers of LGI do not differ. Both of our postprandial studies indicate no clear evidence of a favourable effect of protein intake compared with maltodextrin intake on postprandial responses of ED and LGI. Postprandial responses of some biomarkers of ED and LGI differed between protein sources, but the importance of these differences is not clear, because overall ED and LGI $Z$-scores did not differ between protein sources. Postprandial responses of ED and LGI to the ingestion of maltodextrin did not differ from those after the ingestion of sucrose.

\section{Acknowledgements}

The authors thank Harrie Robins, Jeroen Smets, Sander Offermans, Frank Engel, Thijs Käfer, Floor van den Brand and Imco Janssen for their help with conducting the PROPRES study and second postprandial study. The authors also thank Bas van Bussel for his advice on calculation of the ED and LGI $Z$-scores.

This study was funded by the Top Institute (TI) Food and Nutrition (project number A-1003), Wageningen, The Netherlands. TI Food and Nutrition is a public private partnership of science, industry and government conducting strategic research in food and nutrition (www.tifn.nl).

The authors' responsibilities were as follows - M. A. v. B., E. J. B., J. M. G., C. G. S., P. W. d. L. and S. J. L. B. designed the study; J. D., K. F. M. T.-B. and M. A. v. B. conducted the study; K. F. M. T.-B. and M. A. v. B.: analysed the data; K. F. M. T.-B., M. A. v. B., J. D., E. J. B., J. M. G., P. W. d. L., C. G. S. and S. J. L. B. wrote the manuscript; and M. A. v. B. assumed primary responsibility for the final content. All of the authors read and approved the final version of this manuscript.

None of the authors has any conflicts of interest to declare.

\section{Supplementary material}

For supplementary material/s referred to in this article, please visit http://dx.doi.org/doi:10.1017/S0007114515003530

\section{References}

1. Tielemans SM, Altorf-van der Kuil W, Engberink MF, et al. (2013) Intake of total protein, plant protein and animal protein in relation to blood pressure: a meta-analysis of observational and intervention studies. J Hum Hypertens 27, 564-571.

2. Rebholz CM, Friedman EE, Powers LJ, et al. (2012) Dietary protein intake and blood pressure: a meta-analysis of randomized controlled trials. Am J Epidemiol 176, Suppl. 7, S27-S43.

3. Le Brocq M, Leslie SJ, Milliken P, et al. (2008) Endothelial dysfunction: from molecular mechanisms to measurement, clinical implications, and therapeutic opportunities. Antioxid Redox Signal 10, 1631-1674.

4. Watson T, Goon PK \& Lip GY (2008) Endothelial progenitor cells, endothelial dysfunction, inflammation, and oxidative stress in hypertension. Antioxid Redox Signal 10, 1079-1088.

5. Wong WT, Wong SL, Tian XY, et al. (2010) Endothelial dysfunction: the common consequence in diabetes and hypertension. J Cardiovasc Pharmacol 55, 300-307.

6. Lee H, Lee IS \& Choue R (2013) Obesity, inflammation and diet. Pediatr Gastroenterol Hepatol Nutr 16, 143-152. 
7. Landberg R, Naidoo N \& van Dam RM (2012) Diet and endothelial function: from individual components to dietary patterns. Curr Opin Lipidol 23, 147-155.

8. Lopez-Legarrea P, de la Iglesia R, Abete I, et al. (2014) The protein type within a hypocaloric diet affects obesity-related inflammation: the RESMENA project. Nutrition 30, 424-429.

9. Rebholz CM, Reynolds K, Wofford MR, et al. (2013) Effect of soybean protein on novel cardiovascular disease risk factors: a randomized controlled trial. Eur J Clin Nutr 67, 58-63.

10. Ballard KD, Bruno RS, Seip RL, et al. (2009) Acute ingestion of a novel whey-derived peptide improves vascular endothelial responses in healthy individuals: a randomized, placebo controlled trial. Nutr J 8, 34 .

11. Pal S \& Ellis V (2010) The chronic effects of whey proteins on blood pressure, vascular function, and inflammatory markers in overweight individuals. Obesity (Silver Spring) 18, 1354-1359.

12. Te Morenga LA, Levers MT, Williams SM, et al. (2011) Comparison of high protein and high fiber weight-loss diets in women with risk factors for the metabolic syndrome: a randomized trial. Nutr J $\mathbf{1 0}, 40$.

13. Esser D, Oosterink E, op 't Roodt J, et al. (2013) Vascular and inflammatory high fat meal responses in young healthy men; a discriminative role of IL-8 observed in a randomized trial. PLOS ONE 8, e53474.

14. Esser D, van Dijk SJ, Oosterink E, et al. (2013) A high-fat SFA, MUFA, or n3 PUFA challenge affects the vascular response and initiates an activated state of cellular adherence in lean and obese middle-aged men. J Nutr 143, 843-851.

15. Peairs AD, Rankin JW \& Lee YW (2011) Effects of acute ingestion of different fats on oxidative stress and inflammation in overweight and obese adults. Nutr J 10, 122.

16. Derosa G, D'Angelo A, Salvadeo SA, et al. (2010) Oral glucose tolerance test effects on endothelial inflammation markers in healthy subjects and diabetic patients. Horm Metab Res $\mathbf{4 2}$, $8-13$.

17. Nappo F, Esposito K, Cioffi M, et al. (2002) Postprandial endothelial activation in healthy subjects and in type 2 diabetic patients: role of fat and carbohydrate meals. J Am Coll Cardiol 39, 1145-1150.

18. Mah E, Noh SK, Ballard KD, et al. (2011) Postprandial hyperglycemia impairs vascular endothelial function in healthy men by inducing lipid peroxidation and increasing asymmetric dimethylarginine:arginine. J Nutr 141, 1961-1968.

19. Pal S \& Ellis V (2011) Acute effects of whey protein isolate on blood pressure, vascular function and inflammatory markers in overweight postmenopausal women. Br J Nutr 105, 1512-1519.

20. Teunissen-Beekman KF, Dopheide J, Geleijnse JM, et al. (2012) Protein supplementation lowers blood pressure in overweight adults: effect of dietary proteins on blood pressure (PROPRES), a randomized trial. Am J Clin Nutr 95, 966-971.

21. Teunissen-Beekman KF, Dopheide J, Geleijnse JM, et al. (2013) Blood pressure decreases more after highcarbohydrate meals than after high-protein meals in overweight adults with elevated blood pressure, but there is no difference after 4 weeks of consuming a carbohydrate-rich or protein-rich diet. J Nutr 143, 424-429.

22. Teunissen-Beekman KF, Dopheide J, Geleijnse JM, et al. (2014) Differential effects of proteins and carbohydrates on postprandial blood pressure-related responses. BrJ Nutr $\mathbf{1 1 2}$, 600-608.

23. Dower JI, Geleijnse JM, Gijsbers L, et al. (2015) Supplementation of the pure flavonoids epicatechin and quercetin affects some biomarkers of endothelial dysfunction and inflammation in (pre)hypertensive adults: a randomized double-blind, placebo-controlled, crossover trial. J Nutr (Epublication ahead of print version 15 May 2015).

24. van Bussel BC, Henry RM, Schalkwijk CG, et al. (2011) Fish consumption in healthy adults is associated with decreased circulating biomarkers of endothelial dysfunction and inflammation during a 6-year follow-up. J Nutr 141, $1719-1725$.

25. van Bussel BC, Schouten F, Henry RM, et al. (2011) Endothelial dysfunction and low-grade inflammation are associated with greater arterial stiffness over a 6-year period. Hypertension 58, 588-595.

26. Ridker PM (2003) Clinical application of C-reactive protein for cardiovascular disease detection and prevention. Circulation 107, 363-369.

27. Hirota T, Ohki K, Kawagishi R, et al. (2007) Casein hydrolysate containing the antihypertensive tripeptides Val-Pro-Pro and Ile-Pro-Pro improves vascular endothelial function independent of blood pressure-lowering effects: contribution of the inhibitory action of angiotensinconverting enzyme. Hypertens Res 30, 489-496.

28. Gogebakan O, Kohl A, Osterhoff MA, et al. (2011) Effects of weight loss and long-term weight maintenance with diets varying in protein and glycemic index on cardiovascular risk factors: the diet, obesity, and genes (DiOGenes) study: a randomized, controlled trial. Circulation 124, 2829-2838.

29. Gornik HL \& Creager MA (2004) Arginine and endothelial and vascular health. J Nutr 134, 2880S-2887S; discussion 95S.

30. Mariotti F, Huneau JF, Szezepanski I, et al. (2007) Meal amino acids with varied levels of arginine do not affect postprandial vascular endothelial function in healthy young men. I Nutr 137, 1383-1389.

31. Lin CC, Tsai WC, Chen JY, et al. (2008) Supplements of L-arginine attenuate the effects of high-fat meal on endothelial function and oxidative stress. Int J Cardiol 127, 337-341.

32. Holmer-Jensen J, Karhu T, Mortensen LS, et al. (2011) Differential effects of dietary protein sources on postprandial low-grade inflammation after a single high fat meal in obese non-diabetic subjects. Nutr J 10, 115.

33. Van Bussel BC, Henry RM, Schalkwijk CG, et al. (2012) Lowgrade inflammation, but not endothelial dysfunction, is associated with greater carotid stiffness in the elderly: the Hoorn Study. J Hypertens 30, 744-752.

34. Colacurci N, Chiantera A, Fornaro F, et al. (2005) Effects of soy isoflavones on endothelial function in healthy postmenopausal women. Menopause 12, 299-307. 